\title{
Iron(II) Complexes Featuring a Redox-Active Dihydrazonopyrrole
}

\section{Ligand}

\author{
Kate A. Jesse, ${ }^{[a]}$ Mu-Chieh Chang, ${ }^{[b]}$ Alexander S. Filatov, ${ }^{[a]}$ and John S. Anderson ${ }^{*[a]}$
}

Abstract: Nature uses control of the secondary coordination sphere to facilitate an astounding variety of transformations. Similarly, synthetic chemists have found metal-ligand cooperativity to be a powerful strategy for designing complexes that can mediate challenging reactivity. In particular, this strategy has been used to facilitate two electron reactions with first row transition metals that more typically engage in one electron redox processes. While NNN pincer ligands feature prominently in this area, examples which can potentially engage in both proton and electron transfer are less common. Dihydrazonopyrrole (DHP) ligands have been isolated in a variety of redox and protonation states when complexed to $\mathrm{Ni}$. However, the redox-state of this ligand scaffold is less obvious when complexed to metal centers with more accessible redox couples. Here, we synthesize a new series of Fe-DHP complexes in two distinct oxidation states. Detailed characterization supports that the redoxchemistry in this set is still primarily ligand based. Finally, these complexes exist as 5-coordinate species with an open coordination site offering the possibility of enhanced reactivity.

Coordinated movement of electron and proton transfer during catalysis is vital for productive reactivity in challenging chemical transformations. Second and third row transition metals facilitate an astounding variety of reactions due to their ability to engage in multi-electron and multi-proton elementary steps (i.e. oxidative addition of hydrogen). ${ }^{1}$ However, these metals are often expensive and toxic, providing motivation for the development of catalysts featuring metals such as Fe, which is cheap, largely nontoxic, and widely available. In nature, elegant secondary coordination spheres in enzymatic active sites feature redoxactive cofactors, hydrogen-bonding networks, and proton shuttling pathways which enable difficult transformations with first-row metals. ${ }^{2}$ Using these natural systems as inspiration, many synthetic chemists have worked to develop supporting ligands that can similarly shuttle protons or electrons to substrates. ${ }^{3,4}$ Pincer ligands with an NNN binding pocket feature prominently in this area, particularly as redox non innocent supporting scaffolds. ${ }^{5}$ However, pincer scaffolds which can support both proton and electron transfer are comparatively rarer. ${ }^{6}$

Our laboratory has been exploring this area through the use of dihydrazonopyrrole (DHP) ligand scaffolds. We have found that $\mathrm{Ni}$ complexes of this family do demonstrate reversible ligandbased storage of both protons and electrons as well as engaging in oxidative small molecule reactivity. ${ }^{7}$ The redox-activity of this system is predominantly ligand based as Ni centers, particularly in square planar geometries, have a strong bias for a divalent

[a] University of Chicago Department of Chemistry, $929 \mathrm{E} 57^{\text {th }}$ St. Chicago, IL, 60637

[b] National Taiwan University Department of Chemistry, No. 1 Section 4, Roosevelt Rd, Da'an District, Taipei City, Taiwan 10.

Supporting information for this article is given via a link at the end of the document.((Please delete this text if not appropriate))

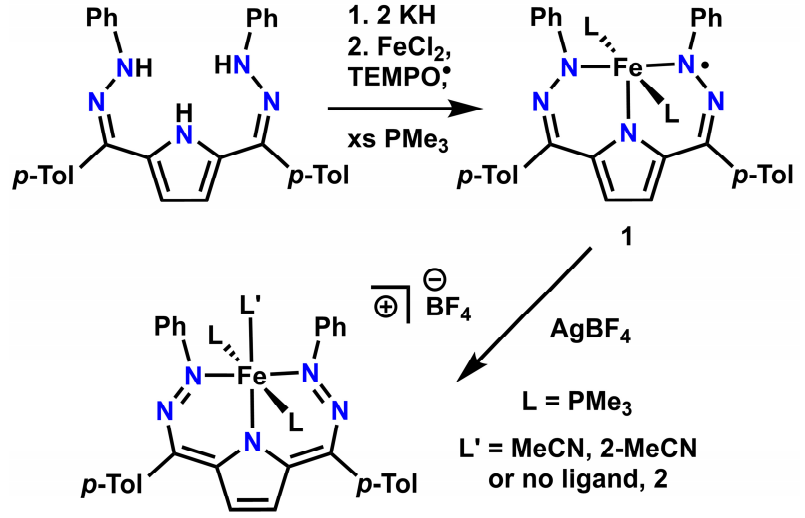

Scheme 1. Synthesis of 1 and 2.

oxidation state. As such, we were interested in exploring complexes where the localization of redox reactions was more ambiguous due to a more redox metal center, namely $\mathrm{Fe}$, which readily populates both $\mathrm{Fe}(\mathrm{II})$ and $\mathrm{Fe}(\mathrm{III})$ oxidation states. Here we report the development of Fe complexes of the ligand ${ }^{\mathrm{Ph}, \mathrm{Tol}} \mathrm{DHP}$ (2,5-bis((2-phenylhydrazono)(p-tolyl)methyl)-pyrrole) in two distinct oxidation states. These complexes adopt 5- or 6coordinate geometries which opens up additional possible sites for substrate binding and reactivity. Unlike the related Ni-system, we have been unable to isolate ligand-hydrogenated complexes with $\mathrm{Fe}$, however, the initial metalation reaction requires the presence of an external $\mathrm{H}$-atom abstractor, suggesting that ligand-based $\mathrm{H}$-atoms are present as reactive intermediates. Most importantly, we have performed detailed characterization of the electronic structure of these complexes revealing that despite the enhanced redox-flexibility of the Fe center, oxidation is still predominantly localized on the supporting pincer ligand. This suggests that Fe complexes could exhibit enhanced reactivity due to the possibility of combined ligand- and metal-based redox reactivity.

The previously reported ${ }^{\mathrm{Ph}, \mathrm{Tol}^{\mathrm{D}} \mathrm{DHP}}$ ligand $^{7 \mathrm{c}}$ was deprotonated with two equivalents of $\mathrm{KH}$ in THF, then filtered and condensed to form a red powder putatively assigned as the doubly deprotonated ligand salt. This powder was added dropwise as a suspension to a 1:1 mixture of $\mathrm{FeCl}_{2}$ and TEMPO in diethylether in the presence of excess $\mathrm{PMe}_{3}$. After work-up, $\left(\mathrm{Ph}^{\text {Tol DHP* }}\right) \mathrm{Fe}\left(\mathrm{PMe}_{3}\right)_{2}(1)$ is obtained as a dark green solid in $31 \%$ crystalline yield (Scheme 1). IR spectroscopy shows no evidence for the presence of $\mathrm{N}-\mathrm{H}$ features suggesting that the additional $\mathrm{H}$ atoms present on ${ }^{\mathrm{Ph}, \mathrm{Tol} D H P}$ have been abstracted as $\mathrm{H}$-atoms by the added TEMPO: While we do not have evidence for any $\mathrm{Fe}-$ containing species with $\mathrm{N}-\mathrm{H}$ functionalities, we note that syntheses carried out in the absence of TEMPO result in a redbrown solid that appears to be a complex mixture of products by NMR spectroscopy. The need for an $\mathrm{H}$-atom abstractor to form 1 suggests that such hydrogenated ligand complexes are likely intermediates, as has been observed in the related Ni systems. ${ }^{7 \mathrm{C}}$

Single crystals of 1 suitable for single-crystal X-ray diffraction (SXRD) were grown from a vapor diffusion of petroleum ether into toluene at room temperature overnight (Figure 1). The SXRD structure of 1 reveals an intermediate geometry between square pyramidal and trigonal bipyramidal $\left(\mathrm{T}_{5}=0.45\right)$. The $\mathrm{Ph}$,TolDHP ligand is bound symmetrically through the beta-N of the hydrazone arms while the two $\mathrm{PMe}_{3}$ ligands are trans to each other. The Fe- $\mathrm{N}_{\text {hydrazone }}$ bond distance averages 1.923(2) A while the $\mathrm{Fe}-\mathrm{N}_{\text {pyrrole }}$ bond distance is $1.937(2) \AA$ (Table 1). These long 

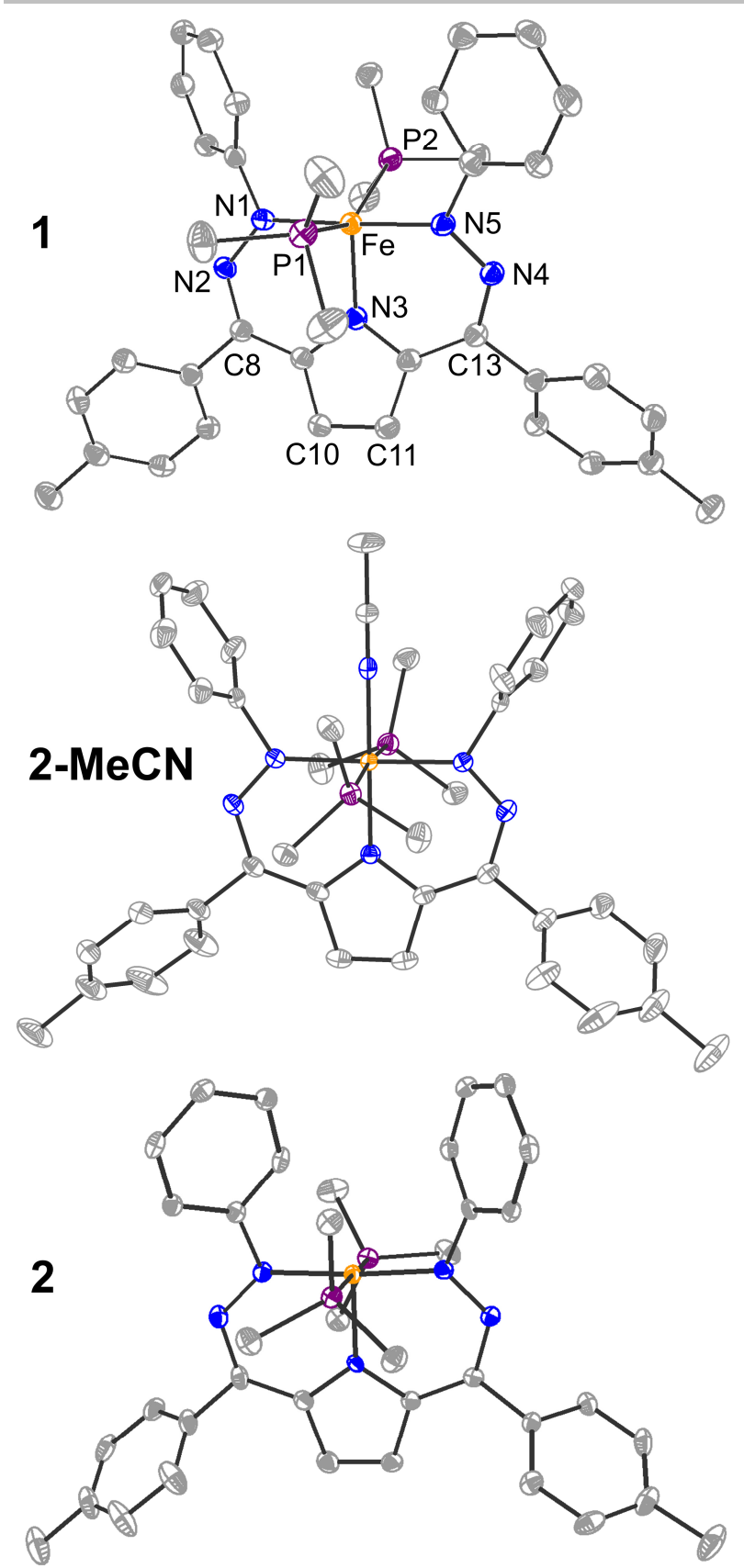

Figure 1. Solid state structures of $1,2-\mathbf{M e C N}$, and 2. Ellipsoids are set to $50 \%$ probability. Hydrogen atoms and counterions have been ommitted for clarity. $\mathrm{Fe}$ is shown in orange, $\mathrm{C}$ in grey, $\mathrm{N}$ in blue, and $\mathrm{P}$ in purple.

Fe-ligand distances are suggestive of a high spin Fe center. Consistent with this hypothesis, the ${ }^{1} \mathrm{H}$ NMR shows four broadened and shifted signals as would be expected for a paramagnetic complex (see SI). Evan's method gives a $\mu_{\text {eff }}$ of 4.6 B.M. consistent with an overall spin of $S=3 / 2$. Similarly, EPR of 1 shows an $S=3 / 2$ rhombic signal featuring $g_{\text {eff }}$ values at $g=5.13$, 2.83 , and 1.81 (see $\mathrm{SI}$ ). This overall spin suggests that the complex is best assigned as either a high spin Fe(II) complex antiferromagnetically coupled to a dianinoic ${ }^{\mathrm{Ph}, \mathrm{Tol}} \mathrm{DHP}$ ligand-based

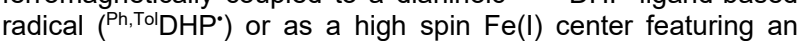
oxidized mono-anionic ligand.

Comparison to Ni complexes featuring the same ${ }^{\mathrm{Ph}, \mathrm{Tol}} \mathrm{DHP}$ ligand in oxidized and radical states (Table 1 ) indicates 1 is best considered an $\mathrm{Fe}$ (II) center antiferromagnetically coupled to a ligand radical. The $\mathrm{C}-\mathrm{C}$ pyrrole backbone, $\mathrm{N}-\mathrm{N}$, and $\mathrm{N}-\mathrm{C}$ bond
Table 1. Selected bond lengths of 1, 2, 2-MeCN, and related Ni complexes (A)

\begin{tabular}{|c|c|c|c|c|c|}
\hline & 1 & $2-\mathrm{MeCN}$ & 2 & $\mathrm{Ni}\left({ }^{\mathrm{Ph}, \mathrm{Tol}} \mathrm{DHP}\right)^{\mathrm{a}}$ & {$\left[\mathrm{Ni}\left({ }^{\mathrm{Ph}, \mathrm{Tol}} \mathrm{DHP}\right)\right]^{+\mathrm{a}, \mathrm{b}}$} \\
\hline $\mathrm{M}^{\mathrm{c}}-\mathrm{N} 1$ & $1.930(2)$ & $1.923(2)$ & $1.875(2)$ & $1.866(2)$ & $1.864(2)$ \\
\hline M-N3 & $1.937(2)$ & $1.916(2)$ & $1.861(2)$ & $1.863(2)$ & $1.860(2)$ \\
\hline M-N5 & $1.916(2)$ & - & $1.861(2)$ & $1.872(2)$ & $1.869(2)$ \\
\hline M-P1 & $2.3980(6)$ & $2.3343(5)$ & $2.3657(9)$ & $2.2319(7)$ & $2.2630(7)$ \\
\hline M-P2 & $2.4219(6)$ & - & $2.3289(9)$ & - & - \\
\hline N1-N2 & $1.351(2)$ & $1.318(2)$ & $1.329(3)$ & $1.342(2)$ & $1.302(2)$ \\
\hline N2-C8 & $1.306(3)$ & $1.345(2)$ & $1.350(3)$ & $1.319(3)$ & $1.348(2)$ \\
\hline N4-C13 & $1.316(3)$ & - & $1.347(4)$ & $1.322(2)$ & $1.342(3)$ \\
\hline N4-N5 & $1.356(3)$ & - & $1.330(3)$ & $1.337(3)$ & $1.314(2)$ \\
\hline C10-C11 & $1.393(3)$ & $1.354(4)$ & $1.351(4)$ & $1.371(3)$ & $1.346(3)$ \\
\hline
\end{tabular}

These complexes also feature a $\mathrm{PMe}_{3}$ ligand. [7c]

${ }^{\mathrm{b}} \mathrm{BF}_{4}$ counterion

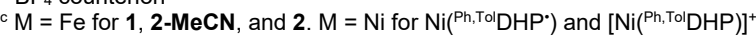

distances most closely align with a ligand radical. This assignment is supported by density functional theory (DFT) calculations. Optimization of 1 was first attempted as a quartet, but resulted in a complex with two unpaired electrons on Fe ferromagnetically coupled to a ligand based radical. This electronic configuration seemed unlikely, so 1 was then optimized with a broken symmetry treatment. This shows spin density on the ligand of opposite character from the spin density on the high-spin $\mathrm{Fe}(\mathrm{II})$ center. Mulliken spin density indicates that 0.72 electrons are localized on ${ }^{\mathrm{Tol}, \mathrm{Ph}} \mathrm{DHP}$, supporting the assignment of a primarily ligand based radical (see $\mathrm{SI}$ ).

Cyclic voltammetry of $\mathbf{1}$ in THF shows a reversible feature at $-0.73 \mathrm{~V}$ and a quasi-reversible feature at $-1.31 \mathrm{~V} v \mathrm{vs}$. $\mathrm{Fc} / \mathrm{Fc}^{+}$ (see SI).These features are similar to those observed in related $\mathrm{Ni}$ systems suggesting that these redox events are ligand based despite the possibility of Fe-based redox chemistry. ${ }^{7}$ Interestingly, there are not any additional redox couples that might be assigned to an Fe-based oxidation within the THF solvent window. Unfortunately, attempts to reduce 1 with a variety of reagents invariably led to a complex mixture of products. However, as might be predicted by the reversibility of the feature at $-0.73 \mathrm{~V} v$ s. $\mathrm{Fc} / \mathrm{Fc}^{+}$, oxidation of $\mathbf{1}$ with $\mathrm{AgBF}_{4}$ in acetonitrile is more tractable and addition of oxidant results in an immediate color change from deep green to dark blue. Further color changes are observed upon workup and drying, and the oxidized complex $\left[(\mathrm{Ph}\right.$, TolDHP $\left.) \mathrm{Fe}\left(\mathrm{PMe}_{3}\right)_{2}\right]\left[\mathrm{BF}_{4}\right](2)$ is isolated as a maroon powder in$91 \%$ yield. Notably, this maroon solid regains a blue color if redissolved in acetonitrile. ${ }^{1} \mathrm{H}$ NMR of 2 in $\mathrm{CD}_{3} \mathrm{CN}$ shows a symmetric diamagnetic species with two bound $\mathrm{PMe}_{3}$ ligands (SI). This indicates that upon oxidation, the $\mathrm{Fe}(\mathrm{II})$ center transitions from a high spin manifold to a low spin manifold. While Fe(II) complexes feature prominently in spin-crossover applications, ${ }^{8}$ examples where spin-crossover can be triggered by redox events are still uncommon. ${ }^{9}$

Single crystals suitable for X-ray diffraction were grown by vapor diffusion of diethylether into concentrated acetonitrile solutions. The SXRD structure obtained from crystals grown in this way shows a pseudo-octahedral geometry, with an acetonitrile adduct in the previously open coordination site trans to the pyrrole $\mathrm{N}$ to give the solvento adduct 2-MeCN (Figure 1). As would be expected for a low spin $\mathrm{Fe}$ (II) center, the $\mathrm{Fe}-\mathrm{N}_{\text {hydrazone }}$ and $\mathrm{Fe}-\mathrm{N}_{\text {pyrrole }}$ distances show a slight contraction from those of 1 to $1.923(1)$ and $1.916(2) \AA$ respectively. Additionally, the pyrrole C-C backbone bond is contracted to 1.354(4) $\AA$, indicative of ligand oxidation (Table 1).

Given that an acetonitrile adduct was observed from the dark blue crystals analyzed by X-ray diffraction, we hypothesized that the color changes observed upon drying/dissolution may arise from acetonitrile binding. To investigate this, $\mathbf{2}$ was dried, then dissolved in a variety of solvents. It was found that in 
coordinating solvents such as acetonitrile or DMF, the solution turned blue, while in less coordinating solvents, such as THF, the solution turned green. In benzene, $\mathbf{2}$ was sparingly soluble and produced a red solution (see SI). Together, these observations suggested that the color changes for $\mathbf{2}$ were arising from variable solvent coordination. To investigate the possibility of a solventfree 5-coordinate complex, 2 was crystallized from a vapor diffusion of petroleum ether into THF. The SXRD structure obtained from these pale green crystals show a 5-coordinate pseudo-square pyramidal complex $\left(T_{5}=0.04\right)$. The ligand bond distances are a close match for those found in complex 2-MeCN, consistent with an oxidized Ph,TolDHP ligand (Table 1). Furthermore, the $\mathrm{Fe}-\mathrm{N}$ and $\mathrm{Fe}-\mathrm{P}$ distances are similar to 2-MeCN suggesting that in both cases this oxidized complex features a low-spin Fe center. These combined observations suggest that 2 can bind solvents in its open coordination site, but that it maintains a low-spin $\mathrm{Fe}(\mathrm{II})$ electronic structure throughout. Therefore, the change from a high spin Fe(II) center in 1 to a low spin Fe(II) center in $\mathbf{2}$ is a product of ligand oxidation rather than solvent coordination.

All of the data acquired on 1 and 2 support ligand-based oxidation events. However, concretely assigning ligand versus metal-based redox localization is challenging and tyipcally requires multiple orthogonal analyses. To further verify that the oxidation of $\mathbf{1}$ to $\mathbf{2}$ was ligand-based, Fe K-edge X-ray absorption spectroscopy (XAS) was conducted on solid powders of the two complexes. Overlaying the X-ray absorption near edge structure (XANES) spectra of these complexes show that $\mathbf{1}$ and $\mathbf{2}$ have effectively identical K-edge's at $7121.3 \mathrm{eV}$ (Figure 2). This data concretely supports that the oxidation state of $\mathrm{Fe}$ in $\mathbf{1}$ and $\mathbf{2}$ is the same, and that the oxidation is ligand-based.

By UV-visible spectroscopy, 2 dissolved in acetonitrile features two additional absorbances at 644 and $940 \mathrm{~nm}$ as compared to the UV-visible spectrum of 2 in benzene (See SI). Interestingly, these absorbances align reasonably well with absorbances seen in 1 at 616 and $912 \mathrm{~nm}$ respectively. In particular, broad absorbances in the 800 to $1000 \mathrm{~nm}$ range have been seen in related $\mathrm{Ni}$ complexes that feature a DHP ligand radical. ${ }^{8}$ The presence of these features may imply that in solution with coordinating solvents such as acetonitrile $\mathbf{2}$ might adopt an electronic structure with a low-spin $\mathrm{Fe}(\mathrm{III})$ metal center antiferromagnetically coupled to a ligand based radical. Notably, in the solid state 2-MeCN shows no evidence for variable temperature redox-changes, as the structures collected at both $100 \mathrm{~K}$ and at room temperature show nearly identical parameters (see SI). To investigate the possibility of a redox tautomerization in solution, XAS was collected of 2-MeCN in a polyethylene glycol matrix with added acetonitrile. However, the XANES spectrum for 2-MeCN prepared in this way shows a similar edge position to that observed for $\mathbf{1}$ and $\mathbf{2}$. These data suggest that the difference in UV-visible spectra between 2-MeCN and $\mathbf{2}$ arises from the changing coordination environment at the Fe center as opposed to a different redox distribution induced by ligand binding.

The data presented here describe a series of $\mathrm{Fe}$ (II) complexes in various redox states. Notably, while Fe should possess an accessible Fe(III) oxidation state, all structural and spectroscopic characterization of the products indicate that these redox events are primarily ligand based. While the Fe oxidation state does not appear to change upon oxidation, the Fe(II) center does undergo a redox-induced spin-crossover from high-spin to low-spin. Finally, the oxidized complex $\mathbf{2}$ displays solvatochromism arising from solvent binding in the open coordination site in this complex. While hydrogenated versions of 1 and 2 have not been isolated, the need for an $\mathrm{H}$-atom abstractor (TEMPO) in the synthesis of 1 suggests that hydrogenated complexes are present as reactive intermediates. Further investigation into the hydrogenative and reductive chemistry of $\mathrm{Fe}$ complexes with DHP ligands is currently underway.

\section{Experimental Section}

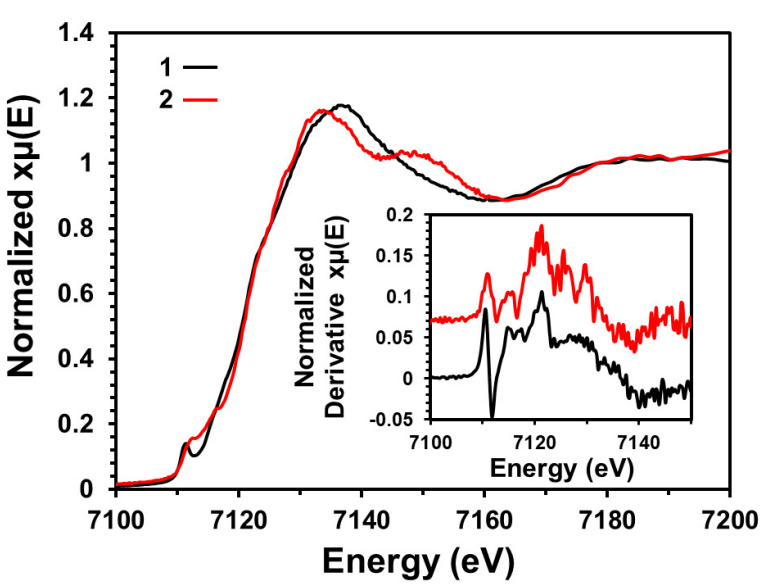

Figure 2. Fe K-edge X-ray absorption spectra of $\mathbf{1}$ and $\mathbf{2}$ as powders. Both samples feature a K-edge inflection point at $7121.3 \mathrm{eV}$. Inset: Derivative spectra of K-edge XAS. 2 is offset for ease of visualization.

\section{General Methods}

All chemicals were purchased from commercial suppliers and used without further purification. All manipulations were carried out under an atmosphere of $\mathrm{N}_{2}$ using standard Schlenk and glovebox techniques. Glassware was dried at $180{ }^{\circ} \mathrm{C}$ for a minimum of two hours and cooled under vacuum prior to use. Solvents were dried on a solvent purification system from Pure Process Technologies and stored over $4 \AA$ molecular sieves under $\mathrm{N}_{2}$. Tetrahydrofuran (THF) and diethyl ether $\left(\mathrm{Et}_{2} \mathrm{O}\right)$ was stirred over NaK alloy and run through an additional alumina column prior to use to ensure dryness. Solvents were tested for $\mathrm{H}_{2} \mathrm{O}$ and $\mathrm{O}_{2}$ using a standard solution of sodium-benzophenone ketyl radical anion. $\mathrm{CD}_{3} \mathrm{CN}, \mathrm{C}_{6} \mathrm{D}_{6}$, and $d_{8}$-toluene were dried over $4 \AA$ molecular sieves under $\mathrm{N}_{2} .{ }^{1} \mathrm{H},{ }^{13} \mathrm{C}\left\{{ }^{1} \mathrm{H}\right\},{ }^{31} \mathrm{P}\left\{{ }^{1} \mathrm{H}\right\},{ }^{19} \mathrm{~F}\left\{{ }^{1} \mathrm{H}\right\}$, and ${ }^{11} \mathrm{~B}\left\{{ }^{1} \mathrm{H}\right\}$ NMR spectra were recorded on Bruker DRX 400 or 500 spectrometers. Chemical shifts are reported in $\mathrm{ppm}$ units referenced to residual solvent resonances for ${ }^{1} \mathrm{H}$ and ${ }^{13} \mathrm{C}\left\{{ }^{1} \mathrm{H}\right\}$ spectra. UV-visible spectra were recorded on a Bruker Evolution 300 spectrometer and analyzed using VisionPro softwar. IR spectra were obtained on a Bruker Tensor II spectrometer with the OPUS software suite. All IR samples were prepared nujol mulls and collected between $\mathrm{KBr}$ plates. EPR spectra were recorded on an Elexsys E500 Spectrometer with an Oxford ESR 900 X-band cryostat and a Bruker Cold-Edge Stinger. EPR data was fit using a least-squares fit in SpinCount. Electrochemical measurements were performed using a BAS Epsilon potentiostat and analyzed using BAS Epsilon software version 1.40.67NT. Cyclic voltammetry measurements were made using a glassy carbon working electrode, platinum wire counter electrode, and silver wire pseudo-reference electrode, and referenced to external $\mathrm{Fc} / \mathrm{Fc}^{+}$. Single crystal X-ray diffraction data were collected in-house using Bruker D8 Venture diffractometer equipped with Mo microfocus X-ray tube $(\lambda=0.71073 \AA)$ or at the Advanced Photon Source of Argonne National Laboratory (beamline 15-ID-B,C,D) using $X$-ray radiation with a wavelength of $\lambda=0.41328 \AA$. X-ray near-edge absorption spectra (XANES) were employed to probe the local environment of Fe. Powder samples were prepared by material grinding finely. A Teflon window was sealed on one side with Kapton tape and powder was then transfer transferred to the inside of this ring before compacting with a Teflon rod and sealing the remaining face with Kapton tape. In the case of RT solution samples, powder was dissolved with minimal solvent, then added to melted polyethylene glycol and mixed well. This was allowed to cool then transfered to a teflon window sealed on one side with Kapton tape. After transfer of the material, the window was sealed with Kapton tape. All sample preparation was performed under an inert atmosphere. Data were acquired at the Advanced Photon Source at Argonne National Labs with a bending magnet source with ring energy at $7.00 \mathrm{GeV}$. Fe K-edge data were acquired at the MRCAT 
MRCAT 9-BM-B,C and 10-BM beam lines. The incident, transmitted and reference $X$-ray intensities were monitored using gas ionization chambers. A metallic iron foil standard was used as a reference for energy calibration and was measured simultaneously with experimental samples. X-ray absorption spectra were collected at room temperature. Data collected was processed using the Demeter software suite ${ }^{10}$ by extracting the EXAFS oscillations $X(k)$ as a function of photoelectron wavenumber $\mathrm{k}$. The theoretical paths were generated using FEFF6 and the models were determined using the fitting program Artemis.

\section{Syntheses}

\section{$\mathrm{Fe}\left(\mathrm{PhDHP}^{\circ}\right)\left(\mathrm{PMe}_{3}\right)_{2}(1)$}

In a $20 \mathrm{~mL}$ vial, $\mathrm{PhDHP}-\mathrm{H}_{2}$ (500 mg, 1 eq.) was dissolved in THF $(10 \mathrm{~mL})$ with stirring. $\mathrm{KH}(83 \mathrm{mg}, 2$ equiv.) were added as a solid and allowed react until all bubbling had stopped ( $\sim 10$ minutes) and the solution was bright red. The solution was then filtered and condensed under vacuum to form a red oil. This was taken up in petroleum ether and then dried under vacuum to form a red powder $\left(\mathrm{PhDHP}-\mathrm{K}_{2}\right)$. In a $20 \mathrm{~mL}$ vial, $\mathrm{FeCl}_{2}(11.3 \mathrm{mg}, 0.09 \mathrm{mmol}$, 1 eq.) was dissolved in diethyl ether $(12 \mathrm{~mL})$ in the presence of excess $\mathrm{PMe}_{3}(0.1 \mathrm{~mL}, 0.9 \mathrm{mmol}, 10$ eq.) and TEMPO $(15.3 \mathrm{mg}$, $0.1 \mathrm{mmol}, 1.1$ eq.) to form a deep read solution. Separately, $\mathrm{PhDHP} \mathrm{K}_{2}$ (50 mg, $0.09 \mathrm{mmol}, 1$ equiv.) was stirred in diethyl ether $(5 \mathrm{~mL})$ to form a suspension. This suspension was added dropwise to form a deep green solution. Once the PhDHP- $\mathrm{K}_{2}$ had been added, the reaction was allowed to stir for 20 minutes, filtered, then condensed under vacuum. This was washed with petroleum ether $(10 \mathrm{~mL})$, taken up in toluene again $(4 \mathrm{~mL})$, filtered, and dried under vacuum. This was taken up in toluene $(4 \mathrm{~mL})$, filtered, then condensed to a concentrated solution. The pure product was obtained by a two-layer crystallization of petroleum ether and the concentrated reaction solution in toluene at $-35^{\circ} \mathrm{C}$. Yield: $31 \%$. Single crystals suitable for XRD were obtained by a vapor diffusion of petroleum ether into a concentrated solution of product in toluene at room temperature overnight. ${ }^{1} \mathrm{H}$ NMR $(400$ $\mathrm{MHz}, d_{8}$-toluene , RT): $\delta=25.49$ (bs), 15.00 (bs), 13.13 (bs), -5.80 (bs). Magnetic Susceptibility: Evans' Method $\left(\mathrm{C}_{6} \mathrm{D}_{6}, \mathrm{RT}, \mu_{\mathrm{B}}\right): \mu_{\text {eff }}=4.6$. EPR experimental $\left(g_{\text {eff }}\right): 5.13,2.83,1.81$. EPR simulated $\left(g_{z}, g_{x}, g_{y}\right)$ : 2.17, 2.28, 1.98. IR (nujol mull, $\mathrm{cm}^{-1}$ ): 2794 (C-H, w), 1583 (s), 1509 (m), $1243(\mathrm{~s}), 1216(\mathrm{~m}), 1096(\mathrm{~s})$. UV-vis, $\mathrm{nm}$ in benzene, $\left(\varepsilon, \mathrm{M}^{-1} \mathrm{~cm}^{-}\right.$ $\left.{ }^{1}\right)$ : 480 (3796), 616 (6612), 690 (5024), 768 (3918), 912 (1018). Anal. Calc 1 + pentane. C, 67.89; H, 7.42; N, 9.21; Found: C, 68.34; H, 6.54; $\mathrm{N}$, 9.98. Note: These combustion results represent the most consistent data we have obtained. Complex $\mathbf{2}$ is unstable and decomposes slowly in the solution or solid state, making obtaining accurate combustion analysis difficult.

\section{$\left[\mathrm{Fe}\left({ }^{\mathrm{Tol}, \mathrm{Ph}} \mathrm{DHP}\right)\left(\mathrm{PMe}_{3}\right)_{2}(\mathrm{MeCN})\right]\left[\mathrm{BF}_{4}\right](2-\mathrm{MeCN})$}

In a $20 \mathrm{~mL}$ vial, 1 (25 mg, $0.04 \mathrm{mmol}, 1$ eq.) was dissolved in acetonitrile (10 mL). Separately, $\mathrm{AgBF}_{4}(7 \mathrm{mg}, 0.04 \mathrm{mmol}, 1$ eq.) was dissolved in acetonitrile $(2 \mathrm{~mL})$, then added to the reaction vial dropwise with stirring to form a deep blue solution. This was allowed to stir for 20 minutes. It was then filtered and condensed under vacuum. The maroon solid was washed with toluene $(5 \mathrm{~mL})$, then taken up in acetonitrile, filtered, then condensed for form a concentrated solution in acetonitrile. One drop of $\mathrm{PMe}_{3}$ was then added, then a vapor diffusion of diethylether into this concentrated solution of product in acetonitrile with excess $\mathrm{PMe}_{3}$ was set up for final purification. Crystals could be obtained overnight at room temperature. Yield: $91 \%$. Single crystals suitable for XRD were obtained by vapor diffusion of diethylether into acetonitrile or by vapor diffusion of petroleum ether into a solution of 2 in THF. ${ }^{1} \mathrm{H}$ NMR $\left(400 \mathrm{MHz}, \mathrm{CD}_{3} \mathrm{CN}\right.$ RT): $\delta=8.12$ (s, 2), 7.67 (d, 4), 2.45 (s, 6), 0.39 (bs, 16). ${ }^{13} \mathrm{C}\left\{{ }^{1} \mathrm{H}\right\}$ NMR $\left(400 \mathrm{MHz}, \mathrm{CD}_{3} \mathrm{CN}, \mathrm{RT}\right): \delta=138.72,138.22,137.07,135.09,131.59$, $129.61,128.97,127.42,125.83,124.78,124.54,20.88,12.66 .{ }^{31} \mathrm{P}\left\{{ }^{1} \mathrm{H}\right\}$ NMR ( $\left.400 \mathrm{MHz}, \mathrm{CD}_{3} \mathrm{CN}, \mathrm{RT}\right): \delta=6.5 .{ }^{19} \mathrm{~F}\left\{{ }^{1} \mathrm{H}\right\} \mathrm{NMR}\left(500 \mathrm{MHz}, \mathrm{CD}_{3} \mathrm{CN}\right.$ $\mathrm{RT}$ ): $\delta$-151.9. ${ }^{11} \mathrm{~B}\left\{{ }^{1} \mathrm{H}\right\} \mathrm{NMR}\left(400 \mathrm{MHz}, \mathrm{CD}_{3} \mathrm{CN}, \mathrm{RT}\right): \delta-1.2$. IR (nujol mull, $\left.\mathrm{cm}^{-1}\right)$ : 2721 (C-H, m), $2669(\mathrm{C}-\mathrm{H}, \mathrm{m}), 2599(\mathrm{C}-\mathrm{H}, \mathrm{w}), 1957(\mathrm{w})$, 1663 (w), $1586(\mathrm{~m}), 1306$ (s), 1283 (s), 1170 (s), 1055 (s), 933 (s). UV-vis, $\mathrm{nm}$ in acetonitrile, $\left(\varepsilon, \mathrm{M}^{-1} \mathrm{~cm}^{-1}\right): 534$ (2661), 644 (4244), 728
(5113), 940 (1135). UV-vis, $\mathrm{nm}$ in acetonitrile, $\left(\varepsilon, \mathrm{M}^{-1} \mathrm{~cm}^{-1}\right): 536$ (2127), 746 (2037), 676 (755). Anal. Calc. $2+\mathrm{MeCN}, \mathrm{C}, 58.84 ; \mathrm{H}, 5.80 ; \mathrm{N}$, 10.29; Found: C, 58.49; H, 5.83; N, 10.23.

\section{Acknowledgements}

This work was supported by the National Institutes of Health (R35 GM133470). We thank the University of Chicago for funding, the $3 \mathrm{M}$ Corporation for a NTFA to J.S.A., and the Sloan Foundation for a Research Fellowship to J.S.A. (FG-2019-11497). We also thank the Research Computing Center at the University of Chicago for providing computing resources. We would like to than Dr. Josh Kurutz for assistance with NMR. Some data reported here were collected at ChemMatCARS Sector 15 which is supported by the NSF under grant number NSF/CHE-1834750. This research used resources of the APS, a U.S. DOE Office of Science User Facility operated for the DOE Office of Science by Argonne National Laboratory under Contract No. DE-AC02-06CH11357. We would like to thank Dr. Yu-Sheng Chen for assistance with SXRD acquisition at 15-ID-B,C,D. We would also like to thank Dr. George Sterbinsky and Dr. Joshua T. Wright for assistance with X-ray absorption spectroscopic data at beamline 9$\mathrm{BM}-\mathrm{B}, \mathrm{C}$ and 10-BM respectively.

Keywords: redox-active ligands - ligand noninnocence - spincrossover $\cdot$ solvochromatism $\cdot$ X-ray absorption spectroscopy

[1] a) R. H. Crabtree, Organometallic Chemistry of the Transition Metals, 6th Edition, 2014 b).Fritz, M.; Schneider, S. In The Periodic Table II: Catalytic, Materials, Biological and Medical Applications; Mingos, D. M. P., Ed.; Springer International Publishing: Cham, 2019; pp 1-36.

[2] a) Liao, Q.; Liu, T.; Johnson, S. I.; Klug, C. M.; Wiedner, E. S.; Morris Bullock, R.; Dubois, D. L. Dalt. Trans. 2019, 48, 4867-4878; b) K. C. Macleod, R. A. Lewis, D. E. Derosha, B. Q. Mercado and P. L. Holland, Angew. Chem. Int. Ed., 2017, 56, 1069-1072.; c) Sinha, S.; Das, S.; Sikari, R.; Parua, S.; Brandaõ, P.; Demeshko, S.; Meyer, F.; Paul, N. D. Inorg. Chem. 2017, 56, 14084-14100;; d) Chirik, P. J. Acc. Chem. Res. 2015, 48 1687-1695.; e) Liu, T.; Liao, Q.; O’Hagan, M.; Hulley, E. B.; DuBois, D. L.; Bullock, R. M. Organometallics 2015, 34, 2747-2764.; f) Wong, J. L.; Sanchez, R. H.; Logan, J. G.; Zarkesh, R. A.; Zillera, J. W.; Heyduk, Chem. Sci. 2013, 4, 1906-1910.; g) Kaim, W. Inorg. Chem. 2011, 50, 9752-9765. h) Helm, M. L.; Stewart, M. P.; Bullock, M. R.; DuBois, M. R.; DuBois, D. L. Science 2011, 333, 863-867.

[3] a) Rauch, M.; Kar, S.; Kumar, A.; Avram, L.; Shimon, L. J. W.; Milstein, D. J. Am. Chem. Soc. 2020, 142, 14513-14521.; b) M. L. Pegis, C. F. Wise, D. J. Martin and J. M. Mayer, Chem. Rev., 2018, 118, 2340-2391.; c) Loewen, N. D.; Thompson, E. J.; Kagan, M.; Banales, C. L.; Myers, T. W.; Fettinger, J. C.; Berben, L. A. Chem. Sci. 2016, 7, 2728-2735.; d) Hill, E. A.; Weitz, A. C.; Onderko, E.; Romero-Rivera, A.; Guo, Y.; Swart, M.; Bominaar, E. L.; Green, M. T.; Hendrich, M. P.; Lacy, D. C.; Borovik, A. S. J. Am. Chem. Soc. 2016, 138, 13143-13146.; e) T. Zell and D. Milstein, Acc. Chem. Res., 2015, 48, 1979-1994.; f) M. Rakowski Dubois and D. L. Dubois, Acc. Chem. Res., 2009, 42, 1974-1982.

[4] a) Luca, O. R.; Crabtree, R. H. Chem. Soc. Rev. 2013, 42, 1440-1459.; b) Chirik, P. J.; Wieghardt, K. Science 2010, 327, 794-795.

[5] a) Joannou, M. V.; Hoyt, J. M.; Chirik, P. J. J. Am. Chem. Soc. 2020, 142, 5314-5330.; b) R. Arevalo and P. J. Chirik, J. Am. Chem. Soc., 2019, 141, 9106-9123.; c) Gardinier, J. R.; Hewage, J. S.; Bennett, B.; Wang, D.; Lindeman, S. V. Organometallics 2018, 37, 989-1000.; d) Bezdek, M. J.; Guo, S.; Chirik, P. J. Science 2016, 354, 730-733.; e) E. J. Thompson and L. A. Berben, Angew. Chem. Int. Ed., 2015, 54, 11642-11646.; f) Heyduk, A. F.; Zarkesh, R. A.; Nguyen, A. I. Inorg. Chem. 2011, 50, 9849-9863.

[6] a) Charette, B. J.; Ziller, J. W.; Heyduk, A. F. Inorg. Chem. 2021, 60, 15791589.; b) T. J. Sherbow, E. J. Thompson, A. Arnold, R. I. Sayler, R. D. Britt, L. A. Berben, Chem. - Eur. J., 2019, 25, 454-458.; c) M. J. Drummond, C. L. Ford, D. L. Gray, C. V. Popescu and A. R. Fout, J. Am. Chem. Soc., 2019, 141, 6639-6650.; d) Rosenkoetter, K. E.; Wojnar, M. K.; Charette, B. J.; Ziller, J. W.; Heyduk, A. F. Inorg. Chem. 2018, 57, 9728-9737.; e) M. B. Ward, A. Scheitler, M. Yu, L. Senft, A. S. Zillmann, J. D. Gorden, D. D. Schwartz, I. Ivanović-Burmazović and C. R. Goldsmith, Nature Chem., 2018, 10, 1207-1212.; f) R. Pramanick, R. Bhattacharjee, D. Sengupta, A. 
Datta and S. Goswami, Inorg. Chem., 2018, 57, 6816-6824.; g) R. Jain, A A. Mamun, R. M. Buchanan, P. M. Kozlowski and C. A. Grapperhaus, Inorg. Chem., 2018, 57, 13486-13493.; h) F. Schneck, M. Finger, M. Tromp and S. Schneider, Chem. Eur. J., 2017, 23, 33-37.; i) G. W. Margulieux, M. J. Bezdek, Z. R. Turner and P. J. Chirik, J. Am. Chem. Soc., 2017, 139, 6110-6113.; j) A. Dauth, U. Gellrich, Y. Diskin-Posner, Y. Ben-David and D. Milstein, J. Am. Chem. Soc., 2017, 139, 2799-2807.; k) B. M. Lindley, Q. J. Bruch, P. S. White, F. Hasanayn and A. J. M. Miller, J. Am. Chem. Soc.

2017, 139, 5305-5308.; I) P. O. Lagaditis, B. Schluschaß, S. Demeshko, C. Würtele and S. Schneider, Inorg. Chem., 2016, 55, 4529-4536.; m) J. T. Henthorn, S. Lin and T. Agapie, J. Am. Chem. Soc., 2015, 137, 1458-1464.; n) T. W. Myers, L. A. Berben, Chem. Sci., 2014, 5, 2771-2777.; o) B. W. Purse, L. H. Tran, J. Piera, B. Åkermark and J. E. Bäckvall, Chem. Eur. J., 2008, 14, 7500-7503.

[7] a) McNeece, A. J.; Jesse, K. A.; Xie, J.; Filatov, A. S.; Anderson, J. S. J. Am. Chem. Soc. 2020, 142, 10824-10832.; b) Chang, M. C.; Jesse, K. A.; Filatov, A. S.; Anderson, J. S. Chem. Sci. 2019, 10, 1360-1367.; c) Chang, M.-C. McNeece, A. J.; Hill, E. A.; Filatov, A. S.; Anderson, J. S. Chem. Eur. J. 2018 24, 8001-8008

[8] a) Batten, S. R.; Bjernemose, J.; Jensen, P.; Leita, B. A.; Murray, K. S.; Moubaraki, B.; Smith, J. P.; Toftlund, H. J. Am. Chem. Soc. 2020, 142, 17670-17680. b) García-Lopez, V.; Waerenborgh, J. C.; Vieira, B. J. C.; Clemente-Leon, M.; Coronado, E., Dalton Trans. 2018, 47, 9156-9163.; c) Shatruk, M.; Phan, H.; Chrisostomo, B. A.; Suleimenova, A. Coord. Chem. Rev. 2015, 289-290, 62-73.; d) Halcrow, M. A. In Spin-Crossover Materials: Properties and Applications; Wiley-Blackwell: Oxford, 2013.; e) Tissot, A.; Shepherd, H. J.; Toupet, L.; Collet, E.; Sainton, J.; Molnar, G.; Guionneau, P.; Boillot, M.-L. Eur. J. Inorg. Chem. 2013, 2013, 1001-1008.; f) Murray, K. S., Eur. J. Inorg. Chem. 2008, 2008, 3101-3121.

[9] a) Govor, E. V.; Al-Ameed, K.; Chakraborty, I.; Coste, C. S.; Govor, O.; Sanakis, Y.; McGrady, J. E.; Raptis, R. G. Angew. Chem. Int. Ed. 2017, 56 582-586.; b) Min, K. S.; DiPasquale, A. G.; Rheingold, A. L.; White, H. S.; Miller, J. S. J. Am. Chem. Soc. 2009, 131, 6229-6236.

[10] a) Ravel, B., Newville, M., ATHENA, ARTEMIS, HEPHAESTUS: data analysis for X-ray absorption spectroscopy using IFEFFIT. J. Synchr. Radn., 2005, 12, 537-541.; b) Newville, M., IFEFFIT: interactive EXAFS analysis and FEFF fitting. J. Synchr. Radn. 2001, 8, 322-324.; c) Rehr, J. J.; Albers R. C. Rev. Mod. Phys. 2000, 72, 621-654 